\title{
Relação entre senso de coerência materno, condições socioeconômicas e percepção da saúde bucal
}

\author{
The relationship between maternal sense of coherence, \\ socioeconomic conditions and the \\ perception of oral health
}

\author{
Valéria Rodrigues de LACERDA ${ }^{1}$ \\ Elenir Rose Jardim Cury PONTES \\ Cecília Lacerda de QUEIROZ²
}

\begin{abstract}
Resumo
O objetivo desta pesquisa foi verificar a relação entre o senso de coerência materno das mães de pré-escolares e as variáveis idade, renda, escolaridade, trabalho, moradia e percepção da saúde bucal. Seiscentas e quarenta mães de pré-escolares do nível III (4 e 5 anos de idade) do município de Campo Grande (Mato Grosso do Sul) responderam a dois questionários, um para a caracterização socioeconômica e percepção da saúde bucal materna e outro para mensurar o senso de coerência materno. Os dados obtidos foram submetidos a análise estatística por meio do programa Bio Estat, versão 5.0. Para a comparação dos escores do senso de coerência materno foi utilizado o Teste Mann Whitney, ao nível de significância de 5\%. Houve associação entre menores valores do senso de coerência materno e condições socioeconômicas desfavoráveis, assim como, em situação de stress, as mães apresentaram uma visão pessimista em relação à própria saúde bucal. Portanto, o senso de coerência materno tem fortes características psicológicas e pode ser um determinante de saúde bucal.
\end{abstract}

Unitermos: Estresse psicológico. Resiliência psicológica. Saúde.

\begin{abstract}
The objective of this study was to evaluate the relationship between the maternal sense of coherence in mothers of preschool children, the variables of age, income, education, work, housing and the perception of oral health. Six hundred forty mothers of Level III preschool children (aged 4 and 5), in the municipality of Campo Grande (Mato Grosso do Sul) responded to two questionnaires: one on their socioeconomic conditions and perception of oral health and the other measuring their maternal sense of coherence. The data was statistically analysed using the BioEstat programmeversion 5.0. We used the Mann-Whitney test to compare the scores for a sense of coherence with a significance level of $5 \%$. Findings suggested an association between a low sense of coherence and adverse socioeconomic conditions, just as how mothers under stressful circumstances expressed a more pessimistic view of their own oral health. The maternal sense of coherence therefore has strong psychological characteristics and may be considered a determinant of oral health.
\end{abstract}

Uniterms: Psychological endurance. Psychological Stress. Health.

$\boldsymbol{\nabla \nabla \nabla v}$

1 Universidade Federal de Mato Grosso do Sul, Faculdade de Odontologia Prof. Albino Coimbra Filho. Av. Senador Filinto Müller, s/n., Vl. Ipiranga, Caixa Postal 549, 79080-190, Campo Grande, MS, Brasil. Correspondência para/Correspondence to: V.R. LACERDA. E-mail: <val99@terra.com.br>.

2 Universidade Católica Dom Bosco, Curso de Psicologia. Campo Grande, MS, Brasil. 
A teoria salutogênica foi proposta por Antonovsky em 1979 (saluto=saúde; gênese=origem) (Antonovsky, 1979), na busca pela superação da dicotomia entre saúde e doença, de acordo com o ideário da Promoção da Saúde contemporânea. Como complemento à teoria patogênica, a salutogênese propõe-se a explicar que os fatores que promovem a saúde são distintos daqueles que modificam o risco para doenças específicas (Lindström, 2001).

Na abordagem salutogênica, pensar a saúde num contexto mais amplo significa reconhecer que ela é resultado da capacidade adaptativa do ser humano ao stress. Além disso, a teoria procura também compreender como os indivíduos conseguem administrar sua vida apesar das condições adversas (Lindström \& Eriksson, 2005).

Antonovsky (1923-1994), o pai da salutogênese e professor de sociologia médica na Universidade de Bem Gurion de Negev, Beersheba, Israel, estudou mulheres no climatério, que viveram em campos de concentração na Segunda Guerra Mundial, constatando que algumas delas mantinham bom estado de saúde físico e mental. Essa constatação despertou seu interesse em aprofundar os estudos, nos quais concluiu que as pessoas que passam por grandes dificuldades e conseguem manter sua saúde física e mental possuem uma característica comum: assumir uma postura mais positiva diante das dificuldades vividas e adaptar-se melhor às situações de stress (Teixeira, 2006).

O elemento central da teoria salutogênica é o Senso de Coerência (SOC), que está relacionado ao bemestar mental. O SOC é um instrumento composto de três variáveis: compreensibilidade (capacidade de compreender um evento), maneabilidade (percepção do potencial de manipulá-lo ou resolvê-lo) e significância (significado que se dá a esse evento) (Bonanato et al., 2007).

O soc implica, fundamentalmente, uma orientação global no sentido de ver a vida estruturada, manejável e com sentido emocional. Trata-se de uma forma individual de pensar, sentir e agir com uma autoconfiança que leva as pessoas a identificarem, beneficiaremse, utilizarem e reutilizarem os recursos disponíveis (Freire, Sheiham \& Hardy, 2001; Pattusi, Hardy \& Sheiham, 2006; Watt, 2002). Estrutura-se a partir do contexto

204 histórico-cultural individual e coletivo e consiste em um determinante importante da posição do indivíduo na contínua alternância entre presença e ausência de bem-estar, o que permite enfrentar as dificuldades da vida como suportáveis e gera um impacto positivo sobre seu estado de tranquilidade (Teixeira, 2006).

No intuito de quantificar e, consequentemente, qualificar o SOC, um questionário testado e validado, numa versão curta com 13 perguntas para serem respondidas em escala Likert, foi proposto por Antonovsky (Teixeira, 2006). Algumas versões em português foram testadas, na busca por propriedades psicométricas satisfatórias em face das diferenças sociais, econômicas e culturais da população brasileira. Assim, no Brasil, uma proposta de transadaptação cultural da versão foi validada, mostrando-se consistente e confiável para a população urbana de mães em diferentes condições sociais (Bonanato et al., 2009a).

Diversos estudos realizados revelaram a relação entre SOC e condições socioeconômicas (Bernabé et al., 2009a; Bernabé et al., 2009b; Lantz et al., 2001; Nilsson, Holmgren \& Westman 2000), assim como entre SOC e percepção de saúde bucal (Hakansson et al., 2003; Nilsson et al., 2000; Savolainen et al., 2005). No Brasil, dois estudos foram realizados sobre a relação do SOC materno com a saúde bucal do filho, um entre adolescentes (Freire et al., 2001) e outro entre pré-escolares (Bonanato et al., 2009b).

Diante do exposto, o SOC pode ser um importante instrumento para subsidiar os setores públicos no planejamento e implementação de políticas sociais. Além disso, a interface da teoria salutogênica com a Promoção da Saúde pode contribuir para a mobilização dos indivíduos no processo de enfrentamento das adversidades, o que pode implicar resultados mais favoráveis em termos de saúde (Bonanato et al., 2009b; Freire et al., 2001; Teixeira, 2006).

Nessa linha, o objetivo deste estudo foi verificar o SOC das mães de pré-escolares, tendo como variáveis a idade, renda, escolaridade, trabalho, moradia e percepção da saúde bucal.

\section{Método}

Realizou-se um estudo transversal com mães (ou na ausência destas, responsáveis do sexo feminino) 
de pré-escolares do nível III (4 e 5 anos de idade), matriculados nos Centros de Educação Infantil (CEINF) do município de Campo Grande (MS).

De acordo com os dados da Secretaria Municipal de Educação (SEMED) e da Secretaria Municipal de Assistência Social (SAS), o município de Campo Grande conta com 89 CEINF, distribuídos da seguinte forma: 18 no Distrito Norte, 28 no Distrito Sul, 17 no Distrito Leste e 26 no Distrito Oeste, atendendo a 3216 crianças na faixa etária de interesse deste estudo (Campo Grande, 2009).

A amostra foi calculada através do Programa Epi Info, versão 3.3.2, baseada nos seguintes parâmetros: população total de crianças de 4 e 5 anos dos CEINF, prevalência de 50\% (+/-5\%), efeito de desenho 2 e nível de significância de 5\%. O valor mínimo foi distribuído proporcionalmente entre os quatro distritos sanitários do município, perfazendo uma amostra de 686 mães, que foram selecionadas através de sorteio.

Para a coleta de dados foram utilizados dois questionários: um para a caracterização socioeconômica e percepção da saúde bucal materna e outro para mensurar o SOC. O instrumento foi previamente testado e validado para mensurar o SOC. Constituiu-se de 13 perguntas, respondidas em uma escala de 1 a 5 pontos tipo Likert, na qual o número 1 correspondia ao extremo negativo, e o número 5 ao extremo positivo, variando em escores de 1 a 5 pontos (Bonanato et al., 2009a). Os escores foram somados para obter um valor absoluto correspondente ao SOC materno, podendo variar de 13 a 65 pontos.

Os dados obtidos foram submetidos a análise estatística por meio do programa Bio Estat, versão 5.0. Para a comparação dos escores do SOC foi utilizado o Teste Mann Whitney, ao nível de significância de 5\%.

O estudo foi aprovado pelo Comitê de Ética em Pesquisa da Universidade Federal de Mato Grosso do Sul (CEP/UFMS), sob o protocolo $n^{\circ} 1.423$. Foram seguidos todos os procedimentos normativos e formais, como assinatura do Termo de Consentimento Livre e Esclarecido (TCLE) pelas participantes, autorizações oficiais da Secretaria Municipal de Educação (SEMED), Secretaria Municipal de Assistência Social (SAS) e dos Centros de Educação Infantil do município (CEINF).

\section{Resultados}

Foram avaliadas 640 mães de pré-escolares, visto que não houve o retorno do questionário preenchido por 46 mães. Os resultados referentes à caracterização socioeconômica da amostra estão apresentados nas Tabelas 1 e 2.

A idade das mães variou entre 18 e 57 anos, com $M=30,1, D P=6,6$ anos e mediana de 29 anos. A maioria tinha um ou dois filhos, predomínio de boa escolaridade (fundamental completo ou acima desse nível) e condição socioeconômica favorável em alguns aspectos (trabalho remunerado, casa própria, com 4 a 6 cômodos), apesar de a renda familiar per capita estar no patamar de até 1⁄2 salário-mínimo (Tabelas 1 e 2).

Tabela 1. Número e porcentagem de mães segundo a caracterização socioeconômica (Parte I) ( $n=640)$. Campo Grande (MS), 2009.

\begin{tabular}{|c|c|c|}
\hline Variáveis & $n$ & $\%$ \\
\hline \multicolumn{3}{|l|}{ Idade (em anos) } \\
\hline Sem informação & 45 & 7,0 \\
\hline 18 a 20 anos & 9 & 1,4 \\
\hline 21 a 30 anos & 339 & 53,0 \\
\hline 31 a 40 anos & 198 & 30,9 \\
\hline 41 a 50 anos & 43 & 6,7 \\
\hline 51 a 57 anos & 6 & 0,9 \\
\hline \multicolumn{3}{|l|}{ Número de filhos } \\
\hline Sem informação & 3 & 0,5 \\
\hline 1 & 157 & 24,5 \\
\hline 2 & 227 & 35,5 \\
\hline 3 & 143 & 22,3 \\
\hline 4 & 72 & 11,3 \\
\hline 5 & 24 & 3,8 \\
\hline Mais de 5 & 14 & 2,2 \\
\hline \multicolumn{3}{|l|}{ Escolaridade da mãe } \\
\hline Sem informação & 3 & 0,5 \\
\hline Analfabeto & 9 & 1,4 \\
\hline Ensino fundamental incompleto & 42 & 6,6 \\
\hline Ensino fundamental completo & 189 & 29,5 \\
\hline Ensino médio incompleto & 124 & 19,4 \\
\hline Ensino médio completo & 120 & 18,8 \\
\hline Curso superior incompleto & 144 & 22,5 \\
\hline Curso superior & 9 & 1,4 \\
\hline \multicolumn{3}{|l|}{ Trabalha fora } \\
\hline Sem informação & 1 & 0,2 \\
\hline Não & 148 & 23,1 \\
\hline $\operatorname{sim}$ & 491 & 76,7 \\
\hline
\end{tabular}


Tabela 2. Número e porcentagem de mães segundo a caracterização socioeconômica (Parte II) (n=640). Campo Grande (MS), 2009.

\begin{tabular}{|c|c|c|}
\hline Variáveis & $\mathrm{n}$ & $\%$ \\
\hline \multicolumn{3}{|l|}{ Moradia } \\
\hline Própria & 322 & 50,3 \\
\hline Própria em aquisição & 37 & 5,8 \\
\hline Alugada & 167 & 26,1 \\
\hline Cedida & 93 & 14,5 \\
\hline Outros & 21 & 3,3 \\
\hline \multicolumn{3}{|l|}{ Cômodos na casa } \\
\hline Sem informação & 12 & 1,9 \\
\hline 1 a 3 & 156 & 24,4 \\
\hline $4 a 6$ & 429 & 67,0 \\
\hline 7 a 9 & 40 & 6,3 \\
\hline 10 ou mais & 3 & 0,5 \\
\hline \multicolumn{3}{|c|}{ Renda familiar per capita (em salário-mínimo) } \\
\hline Sem informação & 37 & 5,8 \\
\hline Até $1 / 2$ salário & 432 & 67,5 \\
\hline$>1 / 2$ a 1 salário & 143 & 22,3 \\
\hline$>1$ a 1 1/2 salários & 22 & 3,4 \\
\hline$>1$ 1 1/2 a 2 salários & 5 & 0,8 \\
\hline >2 salários & 1 & 0,2 \\
\hline
\end{tabular}

O escore no SOC das mães variou entre 13 e 59 pontos, $\mathrm{M}=33,28, \mathrm{DP}=9,39$ pontos e mediana de 33 pontos. Observou-se menor média de $\mathrm{SOC}$ materno nas seguintes condições: 3 filhos ou mais, menor escolaridade, moradia não própria, no máximo 3 cômodos na residência, e renda familiar per capita inferior a 1 1/2 salário-mínimo. Simultaneamente, observou-se que mães com menor média do SOC não tinham uma boa percepção da própria saúde bucal (Tabela 3).

\section{Discussão}

De acordo com os dados da Tabela 1, a idade das mães variou entre 18 e 57 anos, com média de $M=30,1$, $\mathrm{DP}=6,6$ anos e mediana de 29 anos. Esse resultado é importante, uma vez que o SOC dos indivíduos desenvolve-se ao longo da infância e permanece razoavelmente estável após os 30 anos, quando somente eventos significativos na vida podem modificá-lo (Bonanato

Tabela 3. Média e desvio-padrão dos escores do SOC (Senso de coerência) das mães, segundo variáveis socioeconômicas e a percepção materna da própria saúde bucal (n=640). Campo Grande (MS), 2009.

\begin{tabular}{|c|c|c|c|c|}
\hline Variáveis & Mediana & Média & Desvio-padrão & $p$ \\
\hline \multicolumn{5}{|l|}{ Idade } \\
\hline Até 30 anos & 33 & 32,9 & 8,9 & \multirow{2}{*}{0,687} \\
\hline Acima de 30 anos & 33 & 33,4 & 10,4 & \\
\hline \multicolumn{5}{|l|}{ Filhos } \\
\hline Até 2 filhos & 34 & 33,9 & 9,2 & \multirow{2}{*}{0,038} \\
\hline 3 ou mais filhos & 33 & 32,4 & 9,7 & \\
\hline \multicolumn{5}{|l|}{ Escolaridade } \\
\hline Até fundamental & 31 & 30,8 & 9,1 & \multirow{2}{*}{$<0,001$} \\
\hline Médio ou superior & 34 & 35,2 & 9,2 & \\
\hline \multicolumn{5}{|l|}{ Trabalha fora } \\
\hline Sim & 33 & 33,4 & 9,3 & \multirow{2}{*}{0,554} \\
\hline Não & 33 & 32,9 & 9,6 & \\
\hline \multicolumn{5}{|l|}{ Casa própria } \\
\hline $\operatorname{Sim}$ & 34 & 34,1 & 9,3 & \multirow{2}{*}{0,008} \\
\hline Não & 32 & 32,2 & 9,4 & \\
\hline \multicolumn{5}{|l|}{ Cômodos na residência } \\
\hline Até 3 cômodos & 32 & 31,9 & 9,5 & \multirow{2}{*}{0,048} \\
\hline 3 ou mais cômodos & 33 & 33,7 & 9,3 & \\
\hline \multicolumn{5}{|l|}{ Renda familiar per capita } \\
\hline Até 1⁄2 salário-mínimo & 33 & 32,2 & 9,1 & \multirow{2}{*}{$<0,001$} \\
\hline Acima de 1/2 salário-mínimo & 34 & 35,6 & 9,4 & \\
\hline \multicolumn{5}{|c|}{ Percepção materna da própria saúde bucal } \\
\hline Boa & 34 & 34,5 & 9,5 & \multirow{2}{*}{0,005} \\
\hline Não boa & 33 & 32,4 & 9,3 & \\
\hline
\end{tabular}

206

Teste Mann Whitney, ao nível de significância de 5\%. 
et al., 2009b; Geyer, 1997; Silva, 2009). Não houve diferença dos valores medianos do SOC em relação às faixas etárias estudadas (até 30 anos; acima de 30 anos).

O SOC não é considerado um traço da personalidade, mas uma orientação do indivíduo frente à vida e de como enfrentá-la em situações adversas (Larsson \& Kallenberg, 1996). Para o cálculo do SOC materno, utilizou-se uma escala de cinco pontos Likert adaptada, mostrada válida e consistente em estudo realizado com mães de pré-escolares em Belo Horizonte (MG) (Bonanato et al., 2009b). Cada pergunta é pontuada por um escore que ao final são somados. Quanto maior o escore, mais forte o SOC (Hakansson et al., 2003).

OSOC materno variou entre 13 e 59 pontos, com $M=33,28, D P=9,39$ pontos e mediana de 33 pontos. Vários estudos revelam que indivíduos com um SOC forte adaptam-se melhor em situações de stress, uma vez que têm uma visão mais positiva e otimista da vida (Bernabé et al., 2009a; Bonanato et al., 2009a; Nilsson et al., 2000), enquanto pessoas que apresentam altos níveis de stress tendem a apresentar um SOC fraco (Suresky, Zauszniewski \& Bekhet 2008).

Conforme mencionado, a relação entre o SOC materno e as variáveis número de filhos, escolaridade, moradia e renda per capita apresentou um valor do SOC menor nas seguintes condições: 3 filhos ou mais, menor escolaridade, moradia não própria, 3 cômodos na residência e renda familiar per capita inferior a 1/2 salário-mínimo. Os resultados fornecem um forte apoio para uma associação entre o SOC e a condição socioeconômica (Bernabé et al., 2009a; Bernabé et al., 2009b; Gustavsson \& Branholm, 2003; Larsson \& Kallenberg, 1996).

Por outro lado, níveis de renda mais altos estão associados a maior pontuação do SOC (Larsson \& Kallenberg, 1996). Em Israel, em pesquisa realizada com casais de meia idade, verificou-se a associação entre o conjunto das variáveis encontradas em ambientes domésticos e o sentido da coerência na família. Concluiu-se que quanto maior a renda, maior o senso de coerência familiar (Gustavsson \& Branholm, 2003).

Há estudos que confirmam a relação entre SOC e percepção da saúde (Gustavsson \& Branholm, 2003; Nilsson et al., 2000). Uma investigação de base populacional realizada na Suécia estimou o SOC e a percepção da saúde em diferentes estágios de doença no estômago e concluiu que mulheres com tais problemas têm um SOC significativamente menor quando comparadas àquelas que não relataram problemas (Nilsson et al., 2000). Outro trabalho do programa de avaliação de saúde da mulher realizado no Sul da Suécia constatou uma forte relação entre o SOC e a autoavaliação da saúde. As mulheres com um forte SOC avaliaram sua saúde como boa, enquanto aquelas com história de depressão apresentaram SOC fraco (Hakansson et al., 2003).

Vários estudos também comprovam a relação entre o SOC e a saúde bucal (Bonanato et al., 2009b; Dorri, Sheiham, Hardy \& Watt, 2010; Freire et al., 2001; Savolainen et al., 2005). Em Goiânia (GO), 664 adolescentes de escolas públicas e privadas e suas respectivas mães responderam a questionários especificamente desenvolvidos para esse inquérito. A respeito dos adolescentes, foram levantadas informações sobre variáveis demográficas, comportamentais e atitudes em relação à saúde bucal (dieta, higiene bucal, uso de flúor e atendimento odontológico). Das mães, foram abordados aspectos socioeconômicos, dados demográficos, saúde dos filhos desde a infância e os hábitos em relação à saúde bucal materna. Verificou-se uma relação altamente significativa entre o padrão de atendimento odontológico, classe social e educação das mães. Os adolescentes com maior pontuação no SOC e melhor nível social tinham menor probabilidade de ir ao dentista (Freire et al., 2001).

Um levantamento realizado em 2000-2001 pelo Instituto Nacional de Saúde Pública da Finlândia, com adultos dentados de 30-64 anos, verificou a associação entre SOC e o Perfil de Impacto da Saúde Bucal (OHIP), bem como a relação entre os comportamentos de saúde bucal e a condição socioeconômica. Concluiu-se que indivíduos com valores fortes ou moderados do SOC apresentavam um número significativamente menor de problemas de saúde bucal do que aqueles com SOC fraco (Savolainen et al., 2005).

Em Belo Horizonte (MG) foram analisadas 546 mães e seus respectivos filhos, pré-escolares de 5 anos, a fim de verificar a relação entre $\mathrm{SOC}$ materno e a saúde bucal dos filhos. $\mathrm{O}$ estudo demonstrou que filhos de mães com fraco SOC têm maior chance de apresentar cárie dentária do que filhos de mães com forte SOC (Bonanato et al., 2009b). 
Portanto, o SOC tem fortes características psicológicas e pode ser um determinante de saúde bucal (Bonanato et al., 2009b; Savolainen et al., 2005).

\section{Considerações Finais}

Houve associação entre menores valores do SOC e aspectos socioeconômicos desfavoráveis, tais como prole numerosa, baixa escolaridade, baixa renda e moradia pequena e não própria. Nessa situação de stress, as mães apresentaram uma visão pessimista em relação à própria saúde bucal, o que pode indicar uma postura negativa diante das dificuldades vividas.

\section{Referências}

Antonovsky, A. (1979). Health, stress and coping. London: Jossey-Bass.

Bernabé, E., Watt, R. G., Sheiham, A., Suominen-Taipale, A. L., Nordblad, A., \& Savolainen, J. (2009a). The influence of sense of coherence on the relationship between childhood socioeconomic status and adult oral healthrelated behaviours. Community Dentistry Oral Epidemiology, 37 (4), 357-367.

Bernabé, E., Kiwimäki, M., Tsakos, G., Nordblad, A., Savolainen, J., \& Uutela, A. (2009b). The relationship among sense of coherence, socio-economic status, and oral health-related behaviours among finnish dentate adults. European Journal Oral Science, 117 (4), 413-418.

Bonanato, K., Branco, D. B. T., Mota, J. P. T., Ramos-Jorge, M. L., Kaepple, K. C., \& Paiva, S. M. (2009a). Trans-cultural adaptation and psychometric properties of the sense of coherence scale of preschool children. Revista Interamericana de Psicologia, 43 (1), 407-416.

Bonanato, K., Paiva, S. M., Pordeus, I. A., Ramos-Jorge, M. L., Barbabela, D., \& Allison, P. J. (2009b). Relationship between mother's sense of coherence and oral health status of preschool children. Caries Research, 43 (2), 103-109.

Bonanato, K., Scarpelli, A. C., Goursand, D., Mota, J. P. T., Paiva, S. M., \& Pordeus, I. A. (2007). Senso de coerência e experiência de cárie dentária em pré-escolares de Belo Horizonte. Revista Odonto Ciência, 23 (3), 251-255.

Campo Grande. Prefeitura Municipal de Campo Grande. (2009). Relatório de atividades dos centros de educação infantil. Campo Grande: Secretaria Municipal de Assistência Social.

Dorri, M., Sheiham, A., Hardy, R., \& Watt R. (2010). The relationship between sense of coherence and toothbrushing behaviours in Iranian adolescents in Mashhad. Journal of Clinical Periodontology, 37 (1), 46-52.

Freire, M. D. M., Sheiham, A., \& Hardy, R. (2001). Adolescent's sense of coherence, oral health status, and oral healthrelated behaviours. Community Dentistry and Oral Epidemiology, 29 (3), 204-212.
Geyer, S. (1997). Some conceptual considerations on the sense of coherence. Social Science \& Medicine, 44 (12), 1771-1779.

Gustavsson, A., \& Branholm, I. B. (2003). Experienced health, life satisfaction, sense of coherence, and coping resources in individuals living with heart failure. Scandinavian Journal of Occupational Therapy, 10 (3), 138-143.

Hakansson, C., Svartvik, L., Lidfeldt, J., Nerbrand, C., Samsioe, G., \& Schersten, B. (2003). Self-rated health in middle-aged women: associations with sense of coherence and socioeconomic and health-related factors. Scadinavian Journal of Occupational Therapy, 10 (3), 99-106.

Lantz, P. M., Lynch, J. W., House, J. S., Lepkowski, J. M., Mero, R. P., \& Musick, M. A. (2001). Socioeconomic disparities in health change in a longitudinal study of US adults: the role oh health-risk behaviors. Social Science \& Medicine, 53 (1), 29-40.

Larsson, G., \& Kallenberg, K. O. (1996). Sense of coherence, socioeconomic conditions and health. European Journal Oral Science, 6 (3), 175-180

Lindström, B. (2001). O significado de resiliência. Adolescência Latinoamericana, 2 (3), 133-137.

Lindström, B., \& Eriksson, M. (2005). Salutogenesis. Journal of Epidemiology \& Community Health, 59 (6), 440-442.

Nilsson, B., Holmgren, L., \& Westman, G. (2000). Sense of coherence in different stages of health and disease in Northern Sweden. Scandinavian Journal of Primary Health Care, 18 (1), 14-20.

Pattusi, M. P., Hardy, R., \& Sheiham, A. (2006). Neighborhood social capital and dental injuries in Brazilian adolescents. American Journal of Public Health, 96 (8),1462-1468.

Savolainen, J., Suominen-Taipale, A. L., Harju, H. H. P., Uutela, A., Martelin, T., \& Knuuttila, M. (2005). Sense of coherence as a determinant of the oral health-related quality of life: a national study in finnish adults. European Journal of Oral Science, $113(2), 121-127$.

Silva, A. N. (2009). Desvelando os mistérios da saúde bucal: estudo epidemiológico e contribuições da salutogênese para a promoção da saúde bucal. Tese de doutorado não-publicada, Escola Nacional de Saúde Pública Sérgio Arouca, Rio de Janeiro.

Suresky, M. J., Zauszniewski, J. A., \& Bekhet, A. K. (2008). Sense of coherence and quality of life in women family members of the seriously mentally ill. Issues in Mental Health Nursing, 29 (3), 265-78.

Teixeira, K. B. (2006). Senso de coerência materno: relação com a saúde bucal de pré-escolares. Dissertação de mestrado não-publicada, Faculdade de Odontologia, Universidade Federal de Minas Gerais, Belo Horizonte.

Watt, R. G. (2002). Emerging theories into the social determinants of health: implications for oral health promotion. Community Dentistry and Oral Epidemiology, 30 (4), 241-247.

Recebido em: 31/5/2010

Versão final reapresentada em: 30/8/2011

Aprovado em: 14/9/2011 